\title{
ZUBOV'S CONDITION REVISITED
}

\author{
by RONALD A. KNIGHT
}

(Received 10th November 1980, revised 15th December 1981)

\section{Introduction}

Zubov states an elegant necessary and sufficient limit set condition for positive orbital stability of compact invariant sets in his book "Metody A. M. Lyapunova $i$ ih Primenenie" [11]. Stated in terms of our terminology of $L^{-}$for the negative limit set, Zubov's proposition is as follows: $A$ necessary and sufficient condition for positive stability of a compact invariant set $M$ is $L^{-}(X \backslash M) \cap M=\varnothing$. Unfortunately, Zubov's condition $L^{-}(X \backslash M) \cap M=\varnothing$ has subsequently been shown to be necessary but not sufficient (see [9]). Bass and Ura devote considerable effort in [2] and [9] to correcting Zubov's proposition and Desbrow obtains additional results principally concerning unstable sets in [6] and [7]. Ura gives his classical corrected prolongational version of Zubov's assertion on locally compact phase spaces in [9] and extends it to any closed invariant set with compact boundary on such spaces in [10]. Ura's theorem using our terminology of $D^{-}$for the negative prolongation is the following: $A$ necessary and sufficient condition for positive stability of a closed invariant set $M$ with compact boundary on a locally compact phase space is $D^{-}(X \backslash M) \cap M=\varnothing$ (or equivalently, $D^{+}(M)=M$ in terms of the positive prolongation). Bhatia and Hajek show in their monographs [3] and [4] that all of the conclusions above remain unchanged for positively invariant sets with compact boundaries on locally compact spaces which, of course, includes as a very restrictive subclass, the invariant sets.

Our objective is to show that Zubov's proposition holds for certain closed positively invariant sets with compact boundaries on locally compact phase spaces and to classify and characterize certain closed sets for which Zubov's condition fails. Bass and Ura consider compact invariant sets whereas we direct our attention to the larger class of closed positively invariant sets. Attraction theory delineated in references [1] through [5] is employed in order to accomplish this task. In particular we introduce the following useful concept. A weak attractor (attractor) $M$ is called recursive provided the net ( $x t), t \in R^{+}$is frequently (ultimately) in $M$ for each $x$ in the region of weak attraction $A_{w}^{+}(M)$ (attraction $A^{+}(M)$ ). This new terminology can be avoided by simply stating the criterion given in the definition, however, we believe the use of the new terminology is justified because the recursive weak attractors form an important subclass of the class of weak attractors. It was Bhatia and Hajek's generalization of the attraction concept that identified this new class of sets. They show in Proposition 8.10 of [4] that a point $x$ is weakly attracted to a set of $M$ if and only if either the net $(x t), t>0$, is frequently in $M$ or else $L^{+}(x) \cap M \neq \varnothing$. The notion of attractors used in the Bass-Ura work are 
characterized by the condition $\varnothing \neq L^{+}(x) \subset M$ for every $x$ in $A^{+}(M)$ where $M$ is compact invariant. The only recursive attractors satisfying these restrictive conditions are compact components of $X$. Of course, positively invariant compact recursive attractors still satisfy the attraction condition imposed by Bass and Ura but many positively invariant closed recursive attractors, even with compact boundaries, do not. That a recursive weak attractor is not necessarily a recursive attractor, attractor, strong attractor, or stable can readily be seen by simple examples.

The trajectory, orbit closure, limit, prolongational limit, and prolongational relations for a dynamical system $(X, \pi)$ are respectively denoted by $C, K, L, J$, and $D$. The positive and negative versions of these relations carry the appropriate superscript. The symbols $M^{0}, \partial M, \bar{M}$, and $X \backslash M$ denote the interior, boundary, closure, and complement of the set $M$, respectively. All basic properties of dynamical system theory used in this paper are presented in detail in references [3], [4], and [5].

Standing hypothesis: Henceforth, we let $(X, \pi)$ be a given flow on a locally compact Hausdorff phase space $X$.

\section{Stable and unstable attractors}

The following theorem is our first correction of Zubov's proposition. Essentially it asserts that Zubov's condition is necessary and sufficient for certain closed sets to be asymptotically stable.

Theorem 1. A closed weak attractor with compact boundary is stable if and only if it is positively invariant and satisfies $L^{-}(X \backslash M) \cap M=\varnothing$.

Proof. Let $M$ be a closed positively invariant weak attractor with compact boundary for which $L^{-}(X \backslash M) \cap M=\varnothing$. We proceed by contradiction showing that $D^{+}(M)=M$. Let $y \in M$ and $x \in D^{+}(y) \backslash M$. Then $K^{+}(x) \cap M=\varnothing$ and $M$ is contained in the open set $X \backslash K^{-}(x)$. Select an open neighbourhood $V$ of $M$ such that $\bar{V} \subset A_{w}^{+}(M) \cap\left(X \backslash K^{-}(x)\right)$ and $\bar{V} \backslash M^{0}$ is compact. Define $T_{z}=\inf \left\{t \in R^{+}: z t \in V\right\}$ for each $z \in A_{w}^{+}(M)$. Evidently, $0 \leqq T_{z}<$ $+\infty$. Also define $T=\sup \left\{T_{z}: z \in \partial V\right\}$. We now show that $T<+\infty$. For $z \in \partial V$ there is a $z t$ in $V$ for some $t<T_{z}+1$. The continuity of $\pi$ yields $T_{p} \leqq t<T_{z}+1$ for each $p$ in some neighbourhood $V_{z}$ of $z$. There is a finite subcover $\left\{V_{z_{1}}, \ldots, V_{z_{k}}\right\}$ of the compact set $\partial V$. For each point $p$ in $\cup\left\{V_{z_{i}}: i=1, \ldots, k\right\}$ we have $T_{p} \leqq \max \left\{T_{z_{i}}+1: i=1, \ldots, k\right\}$. Thus, $T<+\infty$. Next, $V$ was chosen so that $x \in X \backslash \bar{V}$. Let $\left(x_{i}\right)$ be a net in $V$ converging to $y$ and $\left(t_{i}\right)$ be a net in $R^{+}$such that $x_{i} t_{i} \rightarrow x$. As $\left(x_{i} t_{i}\right)$ is ultimately in $X \backslash \bar{V},\left(x_{i}\right)$ and $\left(t_{i}\right)$ can be selected so that $\left(x_{i} t_{i}\right)$ is in $X \backslash \bar{V}$. Since $C^{+}\left(x_{i}\right)$ is connected and $\partial V$ is compact there is a maximum $\tau_{i}$ in $\left[0, t_{i}\right]$ such that $x_{i} \tau_{i} \in \partial V$. Thus, $t_{i}-\tau_{i} \leqq T$ and $x_{i} t_{i}=\left(x_{i} \tau_{i}\right)\left(t_{i}-\tau_{i}\right) \in \partial V[0, T]$. Hence, $x \in \partial V[0, T]$ and $C^{-}(x)$ must meet $\partial V$. This is absurd because $V$ was chosen so that $C^{-}(x) \subset X \backslash \bar{V}$. Consequently, $D^{+}(y) \subset M$, and hence, $D^{+}(M)=M$. According to Ura's Theorem, $M$ is stable.

Conversely, any stable set is positively invariant and, by Ura's Theorem, $D^{+}(M)=M$ so that $D^{-}(X \backslash M)=X \backslash M$. Whence, $L^{-}(X \backslash M) \cap M=\varnothing$ completing the proof of the theorem. 
Corollary 1.1. A closed weak attractor $M$ with compact boundary is stable if and only if $K^{-}(X \backslash M) \cap M=\varnothing$.

Corollary 1.2. A closed weak attractor with compact boundary satisfying Zubov's condition is stable if and only if it is positively invariant.

Corollary 1.3. If a closed weak attractor $M$ with compact boundary satisfies Zubov's condition, then $C^{+}(M)$ is the smallest stable set containing $M$.

Corollary 1.4. A closed recursive weak attractor with compact boundary containing no semi-trajectory is stable if and only if it is positively invariant.

Corollary 1.5. A closed weak attractor with compact boundary containing no complete orbit is stable if and only if it is positively invariant.

If $X$ is not locally compact, then Zubov's condition need not be sufficient for stability even for positively invariant recursive attractors with compact boundary. The succeeding example is of an unstable compact positively invariant global recursive attractor $M$ satisfying Zubov's condition on a nonlocally compact quotient phase space. Note that $M$ also satisfies Ura's prolongational stability condition.

Example 1. We shall define a flow $(X / \sim, \pi / \sim)$ on a nonlocally compact quotient space determined by an equivalence relation $\sim$ defined on a proper subset of $I=[0,1] \times[0,1]$ with usual planar topology. Let $X=\left(\bigcup_{k=0}^{\infty}\left\{2^{-k}\right\} \times[0,1)\right) \cup\{(0,0),(0,1)\}$. Let $\sim$ be the identity equivalence relation on $X \backslash\{(0,0),(0,1)\}$ and let $(0,0)$ and $(0,1)$ be $\sim$ equivalent. Defining a flow $\pi$ on $I$ using an autonomous system of differential equations so that each point of $[0,1] \times\{0,1\}$ is critical and each line segment $\{x\} \times(0,1)$, $0 \leqq x \leqq 1$, is a regular trajectory directed toward $(x, 0)$ is easy. Let $\pi / \sim$ be the flow induced on $X / \sim$ by $\pi$. Then, $X / \sim$ has countably many components $\left\{\left(2^{-k}, y\right) / \sim: 0 \leqq y<1\right\} ; k=0,1,2, \ldots$ consisting of a regular trajectory and a critical point $\left(2^{-k}, 0\right) / \sim$ as well as a component consisting of a single critical point $(0,0) / \sim$. The set $M=\{(x, y) / \sim \in X / \sim: 2 y \leqq x\}$ is a compact positively invariant global recursive weak attractor in $X / \sim$ with $L^{-}(X / \sim \backslash M)=\varnothing$. However, no positively invariant subneighbourhood of the neighbourhood $\{(x, y) / \sim: 2 y<x+1$ or $4 y<2 x+1\}$ of $M$ exists.

In view of Hajek's Theorem 1 of [8] we give a necessary and sufficient condition for stability of a closed weak attractor on a locally compact metric space. A construction similar to the one in the proof of Theorem 1 on neighbourhoods of boundary points can be employed to show that the condition is sufficient.

Theorem 2. Let $X$ be a metric space. A closed weak attractor $M$ is stable if and only if $M$ is positively invariant, $M$ satisfies Zubov's condition, and every point in the boundary of $M$ has a neighbourhood $V$ such that $C^{+}(V) \backslash M$ has compact closure.

We now turn our attention toward sets not satisfying Zubov's condition.

An arbitrary set $M$ is said to be a saddle set if and only if there exists a 
neighbourhood $U$ of $M$ such that every neighbourhood $V$ of $M$ contains a point $x$ with $C^{+}(x) \not \subset U$ and $C^{-}(x) \not U$.

Classically, the saddle set terminology applied only to compact invariant sets. Recently, however, other sets have been considered fruitfully, particularly closed positively invariant sets. We shall classify and characterize positively invariant and arbitrary weak attractors in terms of the saddle set and attraction concepts.

Bhatia and Hajek show that a positively invariant set $M$ is a strong attractor if and only if it is a stable weak attractor [4], $(8.14,8.15)$. Thus, a closed positively invariant weak attractor with compact boundary is not a strong attractor if and only if Zubov's condition fails. In the remaining theorems we consider classes of weak attractors which are saddle sets, bilateral weak attractors, or unilateral weak attractors on locally compact and arbitrary phase spaces. These results include the classification or characterization statements promised earlier. Note that each weak attractor is either stable, a saddle set, or neither and is either a unilateral weak attractor or else a bilateral weak attractor. The six possible combinations of these conditions form mutually exclusive and exhaustive classes of weak attractors. Examples from each class are easily constructed.

Henceforth, we shall denote the region of strong attraction for a set $M$ by $A_{s}^{+}(M)$. Our next theorem characterizes saddle sets and bilatreal weak attractors for the sets addressed by the first theorem.

Theorem 3. Let $M$ be a closed positively invariant weak attractor with compact boundary. Then we have the following:

(a) $M$ is a saddle set if and only if $A_{w}^{+}(M) \backslash M \neq A_{s}^{+}(M) \backslash M$; and

(b) $M$ is a bilateral weak attractor if and only if $A_{w}^{+}(M) \backslash M=A_{w}^{-}(M) \backslash M$.

Proof. First, let $A_{w}^{+}(M) \backslash M \neq A_{s}^{+}(M) \backslash M$. For a point $x$ in $A_{w}^{+}(M) \backslash A_{s}^{+}(M) \cup M$ we can choose a neighbourhood $U$ of $M$ such that every neighbourhood $W$ of $x$ and $T>0$ satisfy $W T R^{+} \not \subset U$. Select $U$ and a neighbourhood $W$ of $x$ so that $U \cap W=\varnothing$. Let $V$ be any neighbourhood of $M$. Contained in $W$ is a neighbourhood $W_{0}$ of $x$ such that $W_{0} T \subset V \cap U$ for some $T>0$. Moreover, there is a $T_{0}>T$ for which $W_{0} T_{0} \notin U$. Thus, $W_{0} T_{0}$ contains a point $z$ such that $C^{+}(z) \not \subset U$ and $C^{-}(z) \not U$. Consequently, $M$ is a saddle set.

Conversely, let $U$ be a closed neighbourhood of $M$ such that $U \backslash M^{0}$ is compact and each neighbourhood $V$ of $M$ contains a point $z$ for which $C^{+}(z) \not \subset U$ and $C^{-}(z) \not U$. Hence, we can select a net $\left(x_{i}\right)$ converging to a point $x$ in $\partial M$ and nets $t_{i} \geqq 0$ and $s_{i} \leqq 0$ such that $\left(x_{i} t_{i}\right)$ and $\left(x_{i} s_{i}\right)$ converge to points $p$ and $q$, respectively, in $\partial U$. Now, $p \in D^{+}(x)$ and $C^{+}(x) \subset M$ so that $p \in J^{+}(x)$. Thus, $\left(x_{i} s_{i}\right)\left(t_{i}-s_{i}\right)=x_{i} t_{i} \rightarrow p$ and $p \in J^{+}(q)$. Consider the extended flow on the one point compactification $X_{0}^{*}$ of the subspace $X_{0}=A_{w}^{+}(M)$ of $X$ and let $M_{0}$ denote $M$ or $M \cup\{\infty\}$ whenever $M$ is compact or noncompact, respectively. Then, $J^{*+}(q) \not M_{0}$ (the superscript * denotes the extension of a relation on $X_{0}$ to $X_{0}^{*}$ ) so that $q$ is in $A_{w}^{*+}\left(M_{0}\right) \backslash M_{0}$ but, by Ahmad's Theorem 3.3 of [1], not in $A_{s}^{*+}\left(M_{0}\right) \backslash M_{0}$. The point $\infty$ is not in either of these sets yielding $A_{w}^{*}\left(M_{0}\right) \backslash M_{0}=A_{w}^{+}(M) \backslash M$ and $A_{s}^{*+}\left(M_{0}\right) \backslash M_{0} \supset A_{s}^{+}(M) \backslash M$ completing the proof of the converse.

Next, let $A_{w}^{+}(M) \backslash M=A_{w}^{-}(M) \backslash M$. In order to show that $M$ is a negative weak 
attractor we need only demonstrate that $M \subset A_{w}^{-}(M)$. Let $x \in M$. If $C^{-}(x) \subset M$, then $x \in C(x) \subset A_{w}^{-}(M)$. Suppose that $C^{-}(x) \not \subset M$. There is a point $x t$ in $A_{w}^{+}(M) \backslash M=A_{w}^{-}(M) \backslash M$ for some $t<0$. Hence, $x \in C(x t) \subset A_{w}^{-}(M)$. We have $M \subset A_{w}^{-}(M)$.

The converse is obvious because any bilateral attractor has equal positive and negative regions of attraction. This completes the proof of the theorem.

The following theorem classifies the weak attractors of Theorem 3.

Theorem 4. Let $M$ be a closed positively invariant weak attractor with compact boundary. Exactly one of the following holds:

(a) $M$ is stable.

(b) $M$ is a saddle set.

(c) $M$ is a nonsaddle set and some point of $M$ is not strongly attracted to $M$.

Proof. Theorems 8.14 and 8.15 of [4] imply that $M$ is stable if and only if $A_{w}^{+}(M)=A_{s}^{+}(M)$. Equivalently, $M$ is unstable if and only if either $A_{w}^{+}(M) \backslash M \neq A_{s}^{+}(M) \backslash M$ or else $M \not A_{s}^{+}(M)$ and $A_{w}^{+}(M) \backslash M=A_{s}^{+}(M) \backslash M$. Thus, by Theorem $3, M$ is unstable if and only if statement (b) or statement (c) holds. The proof is complete.

Corollary 4.1. A closed positively invariant weak attractor with compact boundary is unstable if and only if it is either a saddle set or a nonsaddle set containing a point not strongly attracted to itself.

\section{REFERENCES}

1. S. Ahmad, Strong attraction and classification of certain continuous flows, Math. Systems Theory 5 (1971), 157-163.

2. R. BASS, Zubov's stability criterion, Bol. Soc. Mat. Mexicana 4 (1959), 26-39.

3. N. Bhatia and O. HaJeK, Theory of dynamical systems, Part II (Technical Note BN-606, Univ. of Maryland, 1969).

4. N. Bhatia and O. HaJeK, Local semi-dynamical systems (Lecture Notes in Math., vol. 90, Springer-Verlag, Berlin and New York, 1969).

5. N. Bhatia and G. Szego, Stability Theory of Dynamical Systems (Springer-Verlag, Berlin and New York, 1970).

6. D. DesBrow, On unstable invariant sets, Funcialaj Ekvacioj 13 (1970), 109-126.

7. D. Desbrow, On asymptotically stable sets, Proc. Edinburgh Math. Soc. 17 (1970), 181-186.

8. O. HAJEK, Compactness and asymptotic stability, Math. Systems Theory 4 (1970), 154-156.

9. T. URA, On the flow outside a closed invariant set; stability, relative stability, and saddle sets, Contra. to Diff. Eq. 3 (1964), 249-294.

10. T. Ura, Sur le courant exterieur a une region invariante; Prolongements d'une characteristique et l'order de stabilite, Funcialaj Ekvacioj 2 (1959), 143-200; nouv. edition, 105-143.

11. V. Zubov, Metody A. M. Lyapunova $i$ ih Primenenie, (Izdatel'stvo Leningradskogo Universiteta, Moscow, 1957). English translation: Methods of A. M. Lyapunov and their Application, AEC-tr-4439, 1961.

Northeast Missouri State University 